\title{
session E: melanoma, sarcoma, rare cancer, brain tumours
}

\section{E1* CLINICAL AND HISTOPATHOLOGICAL RISK FACTORS TO PREDICT SENTINEL LYMPH NODE POSITIVITY, DISEASE FREE AND OVERALL SURVIVAL IN CLINICAL STAGE I-II AJCC SKIN MELANOMA: A PROSPECTIVE STUDY}

Mandalà $\mathrm{M}^{1}$, Imberti $G L^{2}$, Piazzalunga $\mathrm{D}^{3}$, Belfiglio $\mathrm{M}^{4}$, Tondini $\mathrm{C}^{1}$, Barberis $\mathrm{M}^{6}$, Marchesi $L^{5}$, Motta $T^{6}$, Poletti $P^{1}$, Bonomi $L^{1}$, Novellino $L^{3}$, Di Biagio $K^{4}$, Ugo Guerra $^{7}$, Labianca $\mathrm{R}^{1}$

${ }^{1}$ Unit of Medical Oncology, Riuniti Hospital, Bergamo, Italy: ${ }^{2}$ Unit of Dermatology, Riuniti Hospital, Bergamo, Italy; ${ }^{3}$ Unit of Surgery, Riuniti Hospital, Bergamo, Italy; ${ }^{4}$ Department of Clinical Pharmacology and Epidemiology, Consorzio Mario Negri Sud, S. Maria Imbaro, Italy; ${ }^{6}$ Unit of Pathology, Riuniti Hospital, Bergamo, Italy; ${ }^{7}$ Unit of Nuclear Medicine

Background: The aim of the study was to investigate if the tumour infiltrating lymphocytes (TIL) are able to predict the sentinel node positivity (SLN), the disease free (DFS) and overall survival (OS) in clinical stage I-II AJCC primary cutaneous melanoma (PCM) through a prospective mono-institutional data base.

Methods: The study included consecutive patients with PCM, all diagnosed, treated and followed up prospectively by a multidisciplinary team. Logistic regression was used to investigate the association between DFS, OS, SLN positivity and Breslow thickness, Clark level, tumor infiltrating lymphocytes (TIL), ulceration, lesion site, gender, regression and age.

Results: From November 1998 to December October 2008, 1251 patients with PCM were referred to the Bergamo Riuniti Hospital. The median age was $51,7 \pm 16,6$ years; $32,2 \%(\mathrm{~N}=393)$ with $>60$ years, male: $45 \%$. Among those 394 patients with primary vertical growth phase (VGP) melanoma and no clinical evidence of metastatic disease who underwent SLN biopsy. In all, 74 of the 394 patients had 1 positive SLN $(18,8 \%)$. In the multivariate analysis, no extremity primary (extremity vs axial $=$ truncal and headh/neck) OR $0.44,95 \%$ CI $0.22-0.89, \mathrm{p}<0.023$ ), TIL (TIL vs no TIL $0.46,95 \% \mathrm{CI} 0.24-0.88, \mathrm{p}<0.02$ ), and thickness ( $>4 \mathrm{~mm}$ vs $1,1-2 \mathrm{~mm}$ OR $25,95 \% \mathrm{CI}$ 4.95-126.32) predicted SLN positivity. A multivariate stepwise analysis confirmed these results. The histologic status of the SLN was the most significant predictor of DFS and OS. Patients with a negative SLN had a 5-year DFS of $75.9 \%$, compared with $35.2 \%$ in patients with a positive SLN $(\mathrm{p}<.0001)$ and a 5 -years OS of $88.7 \%$ versus $42.9 \%$ respectively $(\mathrm{p}<.0001)$.

Conclusions: Our study demonstrate that The absence of TILs predicts SLN metastasis, at multivariate analysis only the SLN positivity predicts DFS and OS

\section{E2* EFFICACY AND TOXICITY OF SORAFENIB MONOTHERAPY IN PATIENTS WITH ADVANCED SOFT TISSUE SARCOMA FAILING ANTHRACYCLINE-BASED CHEMOTHERAPY}

Santoro $A^{1}$, Stroppa $E^{1}$, Secondino $S^{1}$, Marcon $I^{1}$, Zucali $P^{1}$, Quagliuolo $V^{1}$, Comandone $A^{2}$, Basso $U^{3}$, Soto Parra $H^{4}$, Bertuzzi $A^{1}$

${ }^{1}$ Departimento di Oncologia/Ematologia, Istituto Clinico Humanitas, Rozzano (MII); ${ }^{2}$ Dipartimento di Oncologia Medica, Ospedale Gradenigo, Torino; ${ }^{3}$ stituto Oncologico Veneto IOV-IRCCS, Padova; ${ }^{4}$ Oncologia Medica, Ospedale Garibaldi, Catania

Background and Aims: Sorafenib, an orally administered inhibitor of RAF-family serine/threonine kinases and tyrosine kinase receptors, has demonstrated promising results in the treatment of refractory advanced solid tumors, including soft tissue sarcoma (STS), in terms of disease control and toxicity profile. We performed a multicenter phase II study aimed to evaluate the efficacy and safety of sorafenib in patients with advanced STS progressing after anthracycline-based chemotherapy. Methods: Patients with recurrent STS failing at least one line of chemotherapy and no relevant comorbidities were eligible for the study and received sorafenib $400 \mathrm{mg}$ bid until progression or major toxicity. Patients who received the study drug for at least 4 weeks were considered evaluable for statistical analysis. Primary endpoint of the study was the rate of progression free survival (PFS) at 6 months; the overall clinical benefit, i.e. the proportion of patients achieving response (partial, PR, complete, CR) or stable disease (SD) lasting at least 3 months (RECIST criteria) was also evaluated. Toxicity was graded according to the NCI Common Toxicity Criteria V3.0.

Results: Among 74 patients enrolled in the study, at march 30, 200940 and 44 patients were evaluable for response and toxicity, respectively. Most frequent pathologic subtypes were leiomyosarcoma and lyposarcoma. About half of patients received sorafenib as third or subsequent line of treatment. Six-months PFS was observed in 10/ $40(22,7 \%)$ while overall clinical benefit was documented in $24 / 40$ patients (60\%, $\mathrm{CR}=0 \%, \mathrm{PR}=12,5, \mathrm{SD}=47.5$ ). Most frequently reported adverse events were fatigue, anemia, weight loss, diarrhoea, hand-foot syndrome (HFS) and alopecia. Grade 3-4 toxicities included HFS in $15.9 \%$, diarrhoea in $13.6 \%$, anemia in $4,5 \%$ and mucositis in $4.5 \%$.

Conclusion: Sorafenib is associated with antitumor activity and acceptable tolerability in patients with antracycline-refractory STS. Data from the whole patient population will be presented at the meeting

\section{E3* RETROPERITONEAL SARCOMA: RETROSPECTIVE ANALYSIS OF A LARGE SINGLE INSTITUTION SERIES}

Coladonato $\mathrm{M}^{1}$, Mussi $\mathrm{C}^{1}$, Morenghi $\mathrm{E}^{2}$, Bagnoli $\mathrm{P}^{1}$, Pedrazzoli $\mathrm{P}^{3}$, Secondino $\mathrm{S}^{3}$, A. Santoro ${ }^{3}$, Quagliuolo $V^{1}$

${ }^{1}$ Surgical Department; ${ }^{2}$ Statistical Department; ${ }^{3}$ Oncology Department - IRCCS Istituto Clinico Humanitas, Rozzano (MI), Italy

Background: Prognosis of retroperitoneal sarcoma (RPS) is poor, especially if evaluated on the long run. The mainstay of treatment for RPS is surgery, but both quality and efficacy of the surgical resection decrease after multiple recurrences. We analyzed a large single institution series of RPS with the aim to provide information on the natural history of the disease and to identify prognostic factors of outcome. Methods: From 1998 to 2008, 149 patients with RPS were referred to our Institution and underwent surgery for primary $(n=77)$ or recurrent $(n=72)$ disease. Potential prognostic factors including histology, tumor grading, time to last recurrence and multifocality were retrospectively tested by univariable and multivariable analysis. Primary end-points were local disease free survival (LDFS) and disease-specific survival (DSS) from the first recurrence onward.

Results: Median age of the study population was 55 years (range 20-83); 81 were male. Median FU was 33 months (range 6-122). 5-year LDFS and DSS for the whole population were $27,6 \%$ and $86 \%$, respectively. Nineteen patients $(13 \%)$ developed distant metastases (63\% pulmonary; $68 \%$ extrapulmonary). Twenty-six primary tumors $(33,8 \%)$ required multivisceral associated resection (32\% of leiomyosarcoma; $14 \%$ of liposarcoma; $14 \%$ of other histologies). Histology and tumor grading have been identified as independent prognostic factors for LDFS. Leiomyosarcoma required a higher rate of multivisceral resections than liposarcoma, probably due to their infiltrative growth pattern; liposarcoma had a worse local control (5-year LDFS 18\% versus 56\%). Long term survival was similar in the two groups (5-year DSS 87,6\% versus $80,5 \%$ ), likely related to due to the lower rate of distant metastases in the liposarcoma subgroup. Sex and grading predicted DSS. LDFS and DSS became increasingly shorter along with the number of recurrences.

Conclusions: Our data suggest that in RPS liposarcoma a more aggressive surgical policy should be adopted. Recurrent and multifocal tumors should require a multimodality approach because of the reduced chance of radical surgery.

\section{E4* INDUCTION CHEMOTHERAPY WITH CARBOPLATIN AND TAXOL FOLLOWED BY RADIOTHERAPY AND CONCURRENT WEEKLY CARBOPLATIN + TAXOL IN LOCALLY ADVANCED NASOPHARYNGEAL CARCINOMA}

Mario Airoldi ${ }^{a}$, Anna Maria Gabriele ${ }^{b}$, Massimiliano Garzaroc ${ }^{c}$, Luca Raimondo ${ }^{c}$, Fulvia Pedani ${ }^{a}$, Fabio Beatrice ${ }^{e}$, Giancarlo Pecorari ${ }^{c}$ and Carlo Giordano ${ }^{c}$ ${ }^{a}$ Department of Medical Oncology, San Giovanni Battista Hospital, Turin, Italy; ${ }^{b}$ Radiation Therapy Division, San Giovanni Antica Sede Hospital, Turin, Italy: ${ }^{C}$ Physiopathology Department, University of Turin, Italy; ${ }^{d}$ Neuroscience Department, University of Turin, Italy; ${ }^{e}$ ENT Department, San Giovanni Bosco Hospital, Turin, Italy

Background and purpose: in nasopharyngeal carcinoma (NPC), the role of chemotherapy (CHT), remains controversial for the initial management of the disease. Recent trials and meta-analysis highlight that now, concomitant chemo-

radiationtherapy (CRT) appears to be the standard treatment for locally advanced (T2b and more) and/or node positive patients. No phase II trials have investigated a twodrug combination during conventional, non splitted, RT after a complete course of induction CT. Aim of this study is the evaluation of the clinical outcome of neoadjuvant CHT with carboplatin + taxol followed by RT with weekly carboplatin + taxol combination in locally advanced NPC observed in a non endemic population. Materials and methods: patients with locoregionally advanced NPC were treated with three cycles of induction chemotherapy (CHT) with paclitaxel $(175 \mathrm{mg} / \mathrm{m} 2)$ plus carboplatin (AUC 6), followed by paclitaxel $(60 \mathrm{mg} / \mathrm{m} 2)$ plus carboplatin (AUC 1) and concomitant RT (70 Gy).

Results: in 30 patients treated with such protocol, after the completion of induction CHT and CRT we observed the objective response rates of $90 \%$ and $100 \%$, respectively. Treatment tolerability and toxicity were easily controllable. With a median follow-up 
time of 54 months, 3 - and 5-year disease-free survival was $80 \%$ and $73 \%$ and 3 - and 5 year overall survival was $92 \%$ and $83 \%$. Three- and 5-years locoregional control was $94 \%$ and $80 \%$, and 5 -year distant metastases free survival was $85 \%$.

Conclusions: NACT with paclitaxel and carboplatin followed by paclitaxel plus carboplatin and concomitant CRT represents a feasible, efficient treatment for patients with advanced NPC. This regimen ensures an excellent locoregional disease control and overall survival with a low incidence of distant metastases.

\section{E5* MGMT METHYLATION STATUS BETWEEN FIRST SURGERY AND SECOND SURGERY FOR RECURRENCE IN NEWLY DIAGNOSED GLIOBLASTOMA: CLINICAL IMPLICATIONS}

Brandes $A A^{1}$, Franceschi $E^{1}$, Tosoni $A^{1}$, Fioravanti $A^{2}$, Agati $R^{3}$, Andreoli $A^{2}$, Mazzocchi $\mathrm{V}^{1}$, Morandi $\mathrm{L}^{4}$, Bartolini $\mathrm{S}^{1}$, Ermani $\mathrm{M}^{5}$

${ }^{1}$ Medical Oncology Department, Bellaria-Maggiore Hospital, Azienda USL of Bologna, Bologna, Italy; ${ }^{2}$ Neurosurgery Department, Bellaria-Maggiore Hospital, Azienda USL of Bologna, Bologna, Italy; ${ }^{3}$ Neuroradiology Department, Bellaria-Maggiore Hospital, Azienda USL of Bologna, Bologna, Italy; ${ }^{4}$ Pathology Department, Bellaria Hospital, University of Bologna, Bologna, Italy;

${ }^{5}$ Neurosciences Department, Statistic and Informatic Unit, Azienda OspedaleUniversità, Padova, Italy

Background: $M G M T$ promoter methylation status is a prognostic factor in newly diagnosed glioblastoma patients. However, it is not yet clear whether, and if so how, MGMT methylation status may change; nor is it known whether the prognostic role of this epigenetic feature is retained during the disease course.

Methods: A retrospective analysis was made using a database of 614 glioblastoma patients treated prospectively from $01 / 2000$ to $08 / 2008$. We evaluated only patients who met the following inclusion criteria: age $\geq 18$; PS $0-2$; histological diagnosis of glioblastoma at both first and second surgery for recurrence; postoperative treatment consisting of: a) radiotherapy (RT) followed by adjuvant temozolomide (TMZ) until 2005, and b) TMZ concurrent with and adjuvant to RT after 2005; a time interval $\geq 3$ months between first and second surgery.

Results: MGMT status was evaluated at first and second surgery in all 44 patients (M:F 32:12, median age: 49 years, range: $27-67$ years). In 38 patients ( $86.4 \%)$ MGMT promoter status was assessable at both first and second surgery. MGMT methylation status, changed in $37 \%$ of second surgery samples and more frequently in methylated than in unmethylated pts $(61.5 \%$ vs $24 \%, \mathrm{p}=0.03)$. The median survival was significantly influenced only by MGMT methylation status determined at first surgery $(\mathrm{p}=0.04)$.

Conclusion: Significant changes in MGMT methylation status during the course of GBM occur more frequently in MGMT methylated than unmethylated cases. MGMT methylation status determined at first surgery appears to be of prognostic value, however it is not predictive of outcome following second surgery.

\section{E6* THE ITALIAN EXPERIENCE ON THE FEASIBILITY AND SAFETY OF IPILIMUMAB THERAPY IN PRETREATED METASTATIC MELANOMA PATIENTS}

Maresa Altomonte ${ }^{1}$, Paola Queirolo ${ }^{2}$, Alessandro Testori ${ }^{3}$, Paolo Ascierto ${ }^{4}$ Riccardo Danielli ${ }^{1}$, Anna Maria Di Giacomo ${ }^{1}$, Vanna Chiarion Sileni ${ }^{5}$, Michele Del $V$ ecchio $^{6}$, Ruggero Ridolfi ${ }^{7}$ and Michele Maio

${ }^{1}$ Division of Medical Oncology and Immunotherapy; University Hospital of Siena, Istituto Toscano Tumori, Siena; ${ }^{2}$ Dept. Medical Oncology A, National Institute for Cancer Research, Genoa; ${ }^{3}$ Melanoma and Sarcoma Division, European Institute of Oncology, Milano; ${ }^{4}$ Unit of Medical Oncology, Istituto Nazionale Tumori "Fondazione Pascale", Naples: ${ }^{5}$ Medical Oncology Unit, Istituto Oncologico Veneto-IRCCS, Padua; ${ }^{6}$ Medical Oncology Unit 2, Fondazione IRCCS Istituto Nazionale dei Tumori, Milan; ${ }^{7}$ Somatic Cell Therapy Unit, Istituto Scientifico Romagnolo per lo Studio e la Cura dei Tumori, Meldola, Forll

Background: Clinical trials and single institution experiences have shown effective anti-tumor responses in pre-treated metastatic melanoma (MM) patients (pts) receiving the anti-CTLA-4 monoclonal antibody ipilimumab. We report the Italian (7 Institutions) experience on feasibility and safety of ipilimumab treatment of MM patients within a compassionate use program.

Methods: Seventy-two pts (43 males, 29 females), median age 57 (23-80) years, ECOG performance status 0-2, with MM (51 cutaneous, 12 uveal, 2 mucosal, 7 unknown primary), progressing to 3 median (1-5) systemic therapies for metastatic disease initiated ipilimumab treatment between February and September 2008. In the induction phase (IF) pts received ipilimumab (10mg/Kg i.v.) q3 weeks (wks) x 4 cycles after a 12 wks rest, upon physician judgement, pts received maintenance dosing q12 wks. First tumor assessment (TA) per modified World Health Organization criteria was performed at the end of the IF. Adverse Events (AE) were collected according to Common Terminology Criteria for Adverse events version 3.0.

Results: All pts received at least one ipilimumab dose, 43/72 completed the IF; the remaining 29 pts were withdrawn for early disease progression. Twenty out of 43 pts entered the MF. TA performed at the end of the IF showed partial response (PR) in 4 or stable disease in 12 pts with a clinical benefit of $22 \%$. Grade $3 \mathrm{AE}$ were reported in $8 \%$
(6/72) pts (2 diarrhoea, 1 epigastric pain, 1 myocardial infarction, 1 tumor pain, 1 neurological event).

Conclusions: Ipilimumab treatment in heavily pretreated MM pts is feasible and safe, and results in early clinical benefit in a sizeable proportion of pts.

\section{E7 SALVAGE THERAPY WITH BEVACIZUMAB AND FOTEMUSTINE IN RECURRENT HIGH GRADE GLIOMAS}

Roberta Rudà ${ }^{1}$, Elisa Trevisan ${ }^{1}$, Elisabetta Picco ${ }^{1}$, Stefania Greco Crasto ${ }^{2}$, Maria Grazia Fabrini ${ }^{3}$, Valerio Scotti ${ }^{3}$, Manuela Caroli ${ }^{4}$, Ivan Lolli ${ }^{5}$, Daniele Guarneri $^{1}$, Riccardo Soffietti ${ }^{1}$

${ }^{1}$ University and San Giovanni Battista Hospital, Neuro-Oncology, Turin, Italy; ${ }^{2}$ University and San Giovanni Battista Hospital, Radiology, Turin, Italy; ${ }^{3}$ University and S. Chiara Hospital, Radiotherapy, Pisa, Italy; ${ }^{4}$ University, Neurosurgery, Milan, Italy; ${ }^{5}$ University, Medical Oncology, Bari, Italy

Background: Bevacizumab (BV) given in combination with irinotecan has been reported to be active with acceptable toxicity in recurrent malignat gliomas. Few data are available on the combination of $\mathrm{BV}$ and nitrosoureas, that represents the standard cytotoxic option at recurrence.

Materials and Methods: In this ongoing phase II study patients with MG recurrent after standard treatment (surgery, radiation therapy and concomitant/adjuvant temozolomide) are eligible. Bevacizumab was administered on days 1 and 15 at $10 \mathrm{mg} / \mathrm{Kg}$ and Fotemustine (FTM) was administered weekly for 2 consecutive weeks (days 1 and 8 ) at $75 \mathrm{mg} / \mathrm{m} 2$ (induction phase), followed by a 3 weeks rest period. A maintenance therapy was started in non progressive patients, consisting of BV $10 \mathrm{mg} / \mathrm{Kg}$ and FTM $75 \mathrm{mg} / \mathrm{m} 2$ every 3 weeks until progression or unacceptable toxicity. MRI was performed at baseline, at the end of the induction treatment (fifth week) and every 3-4 cycles. The co-primary endpoints are objective response rate (ORR), based on Macdonald's criteria $(\mathrm{CR}+\mathrm{PR})$ and progression-free survival at 6 months (PFS6), with secondary endpoints of safety, time to tumor progression (TTP) and overall survival.

Results: From April 2008 to April 200941 patients were enrolled. The overall response rate $(\mathrm{CR}+\mathrm{PR})$ was $46 \%$ ( $39 \%$ in GBM; $58 \%$ in gr.III gliomas). The PFS-6 was $44 \%$ ( $38 \%$ in GBM; $48 \%$ in gr.III gliomas). The median TTP was of 4 months (range: 1,2-10,3+) (3,3 months in GBM; 5,9 months in gr.III gliomas). The median overall survival was 5,6 months. A significant clinical improvement was seen in $50 \%$ of patients, with steroid reduction in $60 \%$. Adverse events were haematological toxicity: grade 1-2 leukopenia in 5 patients, grade 3-4 in 3; grade 1-2 thrombocytopenia in 9 patients, grade 3-4 in 4, 3 intatumoral asymptomatic microhemorrhages, 1 hypertensive encephalopathy and 1 stroke.

Conclusions: The combination of BV and FTM is an attractive treatment for recurrent high grade gliomas with acceptable toxicity. The correlations of MGMT status, perfusion MRI and response/outcome are ongoing.

\section{E8 TITOLO THE CLINICAL SIGNIFICANCE OF RADIOLOGICALLY DETECTED PULMONARY NODULES (PN) IN PATIENTS WITH LOCALLY CONFINED SOFT TISSUE SARCOMAS (STS)}

Comandone A, Faletti C*, Berno E, Gino GC, Pochettino P, Bianco L, Bergnolo P, Chiadò Cutin S, Boglione A, Oliva C, Dal Canton O, Garetto F, Brach del Prever EM, Inguil M, Giubellino E

Dept. Medical Oncology Ospedale Gradenigo, Dept. Of Radiology ASO CTOMaria Adelaide*, Gruppo Piemontese Sarcomi - Italian Sarcoma Group

Introduction: Following the current protocol of staging of localized STS (see AIOM and ESMO Guidelines), an increasing number of patients with histologically proven STS undergo routine study using lung CT scan. Visualization of indeterminate pulmonary nodules without other metastatic lesions, represents a clinical dilemma regarding the differential diagnosis and the management of the disease if confined or metastatic.

Patients and Methods: Medical records of STS patients who underwent thoracic CT scans between the years 2000 and 2008 were analyzed. Patients with obvious metastatic disease were not included in the analysis.

Patients were identified via the "Gruppo Piemontese Sarcomi Prospective Data Base"

Results: Out of 1212 new patients assessed from 2000 and 2008 we did 782 staging CT scans. 134 cases $(17 \%)$ with indeterminate pulmonary nodules were identified. We stratified the cases by number and size of nodules. CT scan was repeated every 3-4 months for a median follow up of 24 months. At the end of follow up there was no change in the number or volume in $82 \%$ of the patients with a solitary nodule $<1 \mathrm{~cm}$ and in $75 \%$ multiple nodules $<1 \mathrm{~cm}$ of diameter. On the contrary $88 \%$ of cases with single o multiple pulmonary nodules greater than $1 \mathrm{~cm}$ the situation had progressed. Conclusion: In localized STS pulmonary nodes of undeterminated origin are unlikely a metastatic disease if the nodule is $<1 \mathrm{~cm}$. Huger or multiple lesions are more suspect to represent a secondary deposit. Currently we are exploring the role of PET scan to characterize those lesions. 


\section{E9 PROGNOSTIC SIGNIFICANCE OF MGMT IN ANAPLASTIC ASTROCYTOMAS}

Tosoni $A^{1}$, Franceschi $E^{1}$, Ermani $M^{2}$, Poggi $R^{1}$, Pozzati $E^{3}$, Tomasello $C^{1}$, Rubboli $G^{4}$, Benevento $F^{1}$, Tallini $G^{5}$, Brandes $A A^{1}$

${ }^{1}$ Medical Oncology Department, Bellaria-Maggiore Hospital, Azienda USL of Bologna, Bologna, Italy; ${ }^{2}$ Neurosciences Department, Statistic and Informatic Unit, Azienda Ospedale-Università, Padova, Italy; ${ }^{3}$ Neurosurgery Department, Bellaria-Maggiore Hospital, Azienda USL of Bologna, Bologna, Italy; ${ }^{4}$ Neurology Department, Bellaria-Maggiore Hospital, Azienda USL of Bologna, Bologna, Italy; ${ }^{5}$ Pathology Department, Bellaria-Maggiore Hospital, Azienda USL of Bologna, Bologna, Italy

Background: $M G M T$ methylation status has been found to be an important prognostic factor in glioblastoma patients (pts). However, further data on the epigenetic feature are needed before its role in rare diseases such as anaplastic astrocytomas (AA) can be established.

Methods: A retrospective analysis was made on a database of $139 \mathrm{AA}$ pts followed prospectively from $01 / 1995$ and $08 / 2008$. We evaluated only pts who met the following inclusion criteria: age $\geq 18$ years; PS 0-2; histological diagnosis of AA; postoperative radiotherapy (RT) and chemotherapy (CT). MGMT status was determined with methylation specific PCR. The study aim was to evaluate the role of MGMT methylation status in AA. The log-rank test was employed to evaluate the significance of the prognostic variables.

Results: 80 pts (m/f: 46/34, median age: 41 years, range: $18-71$ years) were enrolled. MGMT was assessable in 71 of $80 \mathrm{pts}(88.8 \%)$, being methylated in $30(42.9 \%)$ and unmethylated in $41(57.7 \%)$ pts. Median PFS was 48.6 months (95\%CI: $33.7-63.5)$, being 96 months (95\%CI: 29-163) and 38 months (95\%CI: 18.9-57.2) in MGMT methylated and unmethylated $\mathrm{pts}$, respectively $(\mathrm{p}=0.09)$. At univariate analysis, complete resection $(\mathrm{p}=0.02)$, age $(\mathrm{p}=0.002)$, and KPS $(\mathrm{p}=0.003)$ were significantly correlated with PFS. At multivariate analysis only age remains correlated with PFS $(\mathrm{p}=0.01)$. Median survival (OS) was 93.7 months (95\%CI: 63.5-123.8), being not reached and 77 months (95\%CI: 20-134.2), in MGMT methylated and unmethylated pts, respectively ( $\mathrm{p}=0.03)$. MGMT methylation $(\mathrm{p}=0.03)$, age $(\mathrm{p}=0.0003)$ and KPS $(\mathrm{p}=0.03)$ were significantly correlated with $\mathrm{OS}$ at univariate analysis. At multivariate analysis, age $(\mathrm{p}=0.0002)$ and $M G M T$ methylation $(\mathrm{p}=0.01)$ were correlated with a better OS.

Conclusions: $M G M T$ methylation status is an independent prognostic factor together with age in AA. This datum should provide the background to improve the therapeutic index with temozolomide concurrent with and adjuvant to RT in AA.

\section{E10 INTENSIVE INDUCTION CHEMOTHERAPY IN POOR- PROGNOSIS ADULT PPNET PATIENTS: A SINGLE INSTITUTION EXPERIENCE}

Bertuzzi A, Castagna L, Stroppa E, Quagliolo V, Navarria P, Secondino S, Giordano L, Santoro A

Departimento di Oncologia/Ematologia, Istituto Clinico Humanitas, Rozzano (MI)

Objective and Methods: To evaluate the impact of intensive versus conventional chemotherapy (CT), as induction treatment before high-dose chemotherapy (HDCT) for treatment of poor-prognosis (local advanced, metastatic or relapsed) adult patients with pPNET. Conventional-dose induction CT (CDICT) consisted of 3-5 cycles of ifosfamide/vincristine/epirubicin (IVE) followed by high-dose cyclophosphamide (CY) or etoposide also given for peripheral blood stem cell (PBSC) mobilization. Intensivedose induction CT (IDICT) consisted of 2-5 cycles of IVE alternating 1-3 cycles of carboplatin/CY/ etoposide (mCAPEC) supported by PBSC collected following the first cycle of IVE. Only pts in response or stable disease after induction CT proceeded to HDCT. Various conditioning regimens for HDCT were utilized. In case of local advanced disease, surgery and radiotherapy were performed when feasible in course of treatment.

Results: From 1997 to 2006, 31 consecutive pts (16 CDICT, 15 IDICT) with median age of 26 yrs, were treated at Our Institution. Disease was advanced in $44 \%$, metastatic in $44 \%$, relapsed in $12 \%$. Five pts had radical surgery at diagnosis and were not evaluable for response. Overall response rate (ORR) after CDICT and IDICT were 67\% (CR 8\%, PR 59\%) (CR 6\%, PR 44\%, SD 6\%) and 57\% (CR 28\% PR 29\%), respectively. HDCT was performed in 23 pts $(72 \%)$. At the end of the whole treatment ORR was 58\% (CR 50\%, PR 8\%) and 50\% (CR 43\%, PR $7 \%$ ) in CDICT vs IDICT groups, respectively. With a median follow-up of 14 months (range 2-130), median time to progression (TTP) and median OS were 10 vs 11 months and 15 vs 12 months for CDICT vs IDICT groups, respectively. One transplant-related death occurred.

Conclusions: In our series of adult patients with pPNET the use of intensive CT in the induction phase of treatment did not provide additional benefit over conventional therapy.

\section{E11 THE CALCIUM-BINDING PROTEIN S100A13 CORRELATES WITH AN ANGIOGENIC PHENOTYPE IN MALIGNANT MELANOMA}

Daniela Massi ${ }^{1}$, Clelia Miracco ${ }^{2}$, Annamaria Piscazzi ${ }^{3}$, Milena Paglierani ${ }^{1}$, Alek Kirov ${ }^{4}$, Vasileios Mourmouras ${ }^{2}$, Claudia Di Serio ${ }^{5}$, Francesca Tarantini ${ }^{5}$, Igor Prudovsky ${ }^{4}$, Matteo Landriscina ${ }^{3}$

'Department of Human Pathology and Oncology, University of Florence (Italy); ${ }^{2}$ Department of Human Pathology and Oncology, University of Siena (Italy): ${ }^{3}$ Department of Medical Sciences, Clinical Oncology Unit, University of Foggia (Italy); ${ }^{4}$ Maine Medical Center Research Institute, Scarborough, ME (USA); ${ }^{5}$ Department of Critical Care Medicine and Surgery, Geriatric Medicine Unit, University of Florence (Italy)

Background: In malignant melanomas angiogenesis and metastatization are sustained by the synthesis and release of pro-angiogenic molecules, such as vascular endothelial growth factor (VEGF) and fibroblast growth factors (FGF), and promotion of increased vascular permeability and endothelial cell proliferation. S100A13 is a small calcium $\left(\mathrm{Ca}^{2+}\right)$-binding protein belonging to the $\mathrm{S} 100$ family. This protein is crucial for the release of angiogenic and pro-inflammatory peptides, such as FGF1 and IL1 $\alpha$, suggesting a possible role in neoplastic progression and metastatization. Several members of the S100 family, including S100A4, S100A6, S100A7 and S100A10, are overexpressed in cancer cells, compared to normal cells, and foster more aggressive phenotypes. S100A13 and VEGF-A are up-regulated in human astrocytic gliomas and correlate with microvessel density and tumour grading. Moreover, S100A13 is associated with a more aggressive, invasive phenotype in lung cancer-derived cell lines. Materials and Methods: To determine the involvement of S100A13 in human melanoma, we studied a series of 66 cutaneous melanocytic lesions: common melanocytic nevi $(\mathrm{n}=14)$, atypical or dysplastic nevi $(\mathrm{n}=14)$, RGP $(\mathrm{n}=10)$ and VGP $(n=19)$ melanomas, and metastases $(n=9)$. Main clinical and pathological features, including outcome, Clark's level, histotype, growth phase and mitotic activity were recorded. Blood microvessel density was determined with CD105/endoglin staining; inducible nitric oxide synthase (iNOS), S100A13, FGF1 and VEGF mRNA and proteins were detected by real time PCR and immunostaining, respectively.

Results: The expression of S100A13 was significantly up-regulated in dysplastic nevi and melanomas, compared to benign nevi $(\mathrm{p}=0.014)$. Expression of S100A13 correlated positively with the level of VEGF staining $(\mathrm{p}=0.01)$, but not with FGF1, which was widely expressed in all lesions. S100A13 and VEGF were both associated with CD105/endoglin expression ( $\mathrm{p}=0.007$ and $\mathrm{p}=0.001$ respectively). No correlation was found between S100A13 and iNOS. While VEGF correlated with risk of relapse $(p=0.006)$, S100A13 protein expression was not associated with status at follow up. Conclusion: S100A13 may cooperate with VEGF in supporting an angiogenic phenotype in human melanoma.

\section{E12 EFFICACY OF TRABECTEDIN (ET-743) IN PRETREATED PATIENTS WITH ADVANCED/METASTATIC SOFT TISSUE SARCOMA}

Cianci $\mathrm{G}^{1}$, De Rosa $\mathrm{P}^{2}$, Tonini $\mathrm{G}^{3}$, Narducci $\mathrm{F}^{1}$, Mentuccia $\mathrm{L}^{1}$, Lolli $\mathrm{I}^{4}$, Ercolino $\mathrm{L}^{5}$, Apice $\mathrm{G}^{6}$, D'Aprile $\mathrm{M}^{7}$, Gamucci $\mathrm{T}^{1}$

${ }^{1}$ Medical Oncology, ASL Frosinone; ${ }^{2}$ Medical Oncology Cardarelli Hospital, Napoli; ${ }^{3}$ Medical Oncology Campus Biomedico, Roma; ${ }^{4}$ Medical Oncology Policlinico, Bari; ${ }^{5}$ Medical Oncology Policlinico, Bari; ${ }^{6}$ Department of Medical Oncology of Sarcomas Fondazione Pascale, Napoli; " Medical Oncology ASL Latina

Background: Trabectedin is a tris, tetrahydroisoquinoline alkaloid isolated from the marine ascidian Ecteinascidia turbinata. It is the first of a new class of antitumor agents with a mechanism of action that involves the transcription-dependent nucleotide excision repair system. It has recently received approval, as a second-line therapy, for soft tissue sarcoma (STS). We report the experience of seven Italian center of medical oncology.

Methods: Thirteen patients (pts) with advanced/metastatic STS are included in the analysis. All pts included had failed or progressed on $\geq 1$ lines of chemotherapy ( 4 pts have made 1 previous line, 3 two previous lines, 4 three previous lines and 2 four previous lines). Trabectedin was administered at $1.3 \mathrm{mg} / \mathrm{m}^{2}$ as a 24 -hour IV infusion every 3 weeks with $20 \mathrm{mg}$ of desametason premedication at T -12 and T 0 . Disease assessment was performed every 2-3 courses utilizing RECIST criteria.

Results: Median age was 56 (range 36-72), M/F 7 (53.8\%)/6 (46.2\%), ECOG PS 0/1 was $5(38.5 \%) / 8(61.5 \%)$. Median cycles was 3(range 2-6). Twelve pts are evaluable for response and toxicity, one pts is too early. Partial response was obtained in 5 pts (38.5 $\%$ ) (1 pts liposarcoma, 3 pts myxoid liposarcoma and 1 pts synovial sarcoma); stable disease in $2(15.4 \%$ ) (1 pts liposarcoma and 1 pts leiomyosarcoma) and progression disease in 5 pts (38.5\%) (2 pts leiomyosarcoma, 1 pts liposarcoma, 1 pts myxoid liposarcoma and 1 pts carcinosarcoma).

Hematologic toxicity grade 3 was observed in 2 pts ( 1 pts neutropenia, 1 pts anemia) and grade 2 in 2 pts (anemia). A laboratory transaminase elevation was seen in 7 patients: 1 grade 2 and 6 grade 1 . All hepatic toxicities were transient. Three pts have developed asthenia grade 2 .

Conclusions: Trabectedin is an active drug in advanced pre-treated STS patients. It has an acceptable and manageable toxicity profile. 

E13 POTENTIAL ROLE OF PHOSPHO PROTEIN KINASE B/AKT IN
THE PROGRESSION OF GLIOBLASTOMA

Francesca Maines*, Flavia Miccichè*, Veronica Parolin*, Rocco Pastano*, Nicola Martinelli*, Claudio Ghimenton`, Antonio lannucci^, Gian Luigi Cetto*, Chiara Dell'Agnola*

- Department of Clinical and Experimental Medicine, Section of Oncology, University of Verona, Italy: • Department of Medicine, Division of Hematoncology, Bone Marrow Transplant Unit, European Institute of Oncology; Milan, Italy; *Department of Clinical and Experimental Medicine, Section of Internal Medicine, University of Verona, Italy; "Department of Pathology, Civile Maggiore Hospital, Verona, Italy

Glioblastoma has a poor prognosis with a median overall survival of 12-14 months, despite the multimodal treatment. Therefore, new therapeutic approaches are under investigation, mainly taylored to peculiar targets of the disease. Protein-kinase B (Akt) has been shown to have a pro-survival role also in cancer and it would potentially represent a marker of interest for glioblastoma. We retrospectively evaluated the role of Akt at the diagnosis and at the relapse in a casistic of 42 glioblastoma patients (median age 52), homogeneous for surgical-medical treatment received. Paraffin-embedded tissue samples from patients and from normal autoptic brain were studied by immunoistochemical analysis for total and phosphoAkt expression both at the time of the diagnosis and of the relapse. Akt expression was also correlated to tissue samples morphology including necrosis and vascularization. In clear contrast with normal brain tissue samples found negative both for total and phosphoAkt, all glioblastoma patients at the diagnosis were positive for total Akt with 41/42 of them positive for phosphoAkt. Focusing on this active form of Akt, we observed an increasing trend of it expression at the relapse, with 10 patients becoming strong positive. Interestingly none of the patients did decrease phosphoAkt expression at the time of relapse. High posphoAkt expression at the diagnosis correlated with earlier relapse (median time to progression 11 months in strong positive vs 13.4 months in weak positive patients). This finding was evident also at different time points during the follow-up: at five months after surgery strong positive phosphoAkt patients at the diagnosis relapsed in a double percentage compared to the weak positive subjects. Tumor neovascularization was found highly positive for phoshoAkt. Furthermore, phosphoAkt was increased at the periphery of "pseudopalisade" necrosis, but not in the diffuse form. Our data suggest a critical role of phosphoAkt in the pathogenesis and progression of glioblastoma; however, perspective studies with a larger number of patients are needed to support our findings.

\section{E14 IPILIMUMAB IN THE DAILY PRACTICE: FEASIBILITY, SAFETY, EFFICACY AND LONG-TERM FOLLOW-UP IN HEAVILY PRETREATED METASTATIC MELANOMA PATIENTS}

Anna Maria Di Giacomo ${ }^{1}$, Riccardo Danielli ${ }^{1}$, Luana Calabrò ${ }^{1}$, Erica Bertocci ${ }^{1}$, Chiara Nannicini ${ }^{1}$, Clelia Miracco ${ }^{2}$, Maurizio Biagioli $^{3}$, Maria Antonietta Mazzei ${ }^{4}$, Maresa Altomonte ${ }^{1}$, Michele Maio ${ }^{1,2}$

${ }^{1}$ Medical Oncology and Immunotherapy; ${ }^{2}$ Pathology; ${ }^{3}$ Dermatology; ${ }^{4}$ Radiology, University Hospital of Siena, Istituto Toscano Tumori, Siena (Italy)

Background: Effective anti-tumor responses are being observed in metastatic melanoma (MM) patients (pts) treated with the anti-CTLA-4 antibody ipilimumab in clinical trials; however, no data support the feasibility and clinical effectiveness of ipilimumab use in the daily practice of MM. We report on a single Institution experience utilizing ipilimumab within a compassionate program for MM pts.

Methods: 27 stage III (2) or IV (25) pts (14 males, 13 females), median age 55 (23-77) years, ECOG performance status $0-1$, with MM ( 23 cutaneous, 3 uveal, 1 mucosal) progressing to 3 median (1-5) systemic therapies for metastatic disease received ipilimumab. Eight pts had evidence (6) or history (2) of brain metastases and 11 elevated ( $>1 \mathrm{x}$ upper limit of normal [ULN]) LDH values. In the induction phase (IF) pts received ipilimumab ( $10 \mathrm{mg} / \mathrm{kg}$ i.v.) q3 weeks (wks) for 4 cycles; following a $12 \mathrm{wks}$ rest treatment was repeated q12 wks in the maintenance phase (MF). Tumor assessment (TA) per modified World Health Organization Criteria was performed at baseline, week (wk) $12( \pm 2)$ and wk 24, then every 12 wks. Adverse Events (AE) and immune related AE (irAE) were collected according to Common Terminology Criteria for Adverse Events version 3.0.

Results: All pts received at least one ipilimumab dose, and 21/27 completed the IF; the remaining pts, were withdrawn for $\mathrm{AE}$ severity (3 pts) or disease progression (3 pts).Eleven pts entered the MF. TA at wk 12 showed partial response (PR) in 1/21 o stable disease (SD) in 5/21 pts. TA at wk 24 showed PR and SD in $3 / 11$ and 5/11 pts, respectively, with an ongoing clinical benefit $(\mathrm{SD}+\mathrm{PR}+\mathrm{CR})$ of $30 \%(8 / 27 \mathrm{pts})$; these pt are still on treatment. The median overall survival is 29 wks (20-55). Slow, steady declines in tumor volume and appearance of new lesions with subsequent shrinking of total tumor burden has been observed. One patient experienced Grade $3 \mathrm{AE}$ (myocardial infarction considered unrelated to treatment), 1 patient Grade 4 irAE (pancytopenia) and 2 pts Grade 3 irAE (diarrhea).

Conclusions: Ipilimumab treatment is feasible, safe and clinically effective also in the common daily practice and in heavily pretreated, progressing, MM pts. A sizeable proportion of these pts experiences durable clinical benefit and long-term survival.

\section{E15 IPILIMUMAB IN PRETREATED METASTATIC UVEAL} MELANOMA PATIENTS: SAFETY AND CLINICAL EFFICACY

Riccardo Danielli ${ }^{1}$, Paola Queirolo ${ }^{2}$, Alessandro Testori ${ }^{3}$, Ruth Plummer ${ }^{4}$ Evangelia Razis ${ }^{5}$, Vanna Chiarion Sileni ${ }^{6}$, Luana Calabrò ${ }^{7}$, Anna Maria Di Giacomo $^{8}$, Ruggero Ridolfi ${ }^{9}$, Michele Maio ${ }^{1}$

'Division of Medical Oncology and Immunotherapy; University Hospital of Siena, Istituto Toscano Tumori, Siena, Italy; ${ }^{2}$ Dept. Medical Oncology A, National Institute for Cancer Research, Genoa, Italy; ${ }^{3}$ Melanoma and Sarcoma Division, European Institute of Oncology, Milano, Italy; ${ }^{4}$ Northern Institute for Cancer Research Newcastle university, Newcastle upon Tyne, United Kingdon; ${ }^{5}$ Hygeia Hospital, First Dept. of Medical Oncology, Athens, Greece; ${ }^{6}$ Medical Oncology Unit, Istituto Oncologico Veneto-IRCCS, Padua, Italy; ${ }^{7}$ Somatic Cell Therapy Unit, Istituto Scientifico Romagnolo per lo Studio e la Cura dei Tumori, MeldolaForll, Italy

Background: The anti-CTLA-4 monoclonal antibody ipilimumab induces antitumor responses in cutaneous metastatic melanoma (MM) patients (pts). However, no data are available on the clinical effectiveness of ipilimumab treatment in uveal MM pts. We report the European (7 institutions) experience utilizing ipilimumab in uveal MM pts.

Methods: Fourteen stage IV pts (8 male, 6 female), median age 57 (30-76) years, ECOG performance status $0-1$, with uveal MM progressing to 2 median (1-4) previous therapies for metastatic disease received ipilimumab within a compassionate use program. Thirteen pts had history (1) or evidence (12) of liver metastases, 2 of brain metastases and 3 of elevated ( $>1 \mathrm{x}$ upper limit of normal [ULN]) LDH values. In the induction phase (IF) pts received ipilimumab $(10 \mathrm{mg} / \mathrm{Kg}$ i.v. ) q3 weeks (wks) x 4 cycles; after a 12 wks rest, treatment was repeated q12 wks in the maintainance phase (MF). Tumor assessment (TA) per modified World Health Organization criteria was evaluated at baseline, at week (wk) 12 and wk 24, than every 12 wks. Adverse Events (AE) and immune related AE (irAE) were collected according to Common Terminology Criteria for Adverse events version 3.0

Results: All pts received at least one ipilimumab dose, and 10/14 completed the IF. The remaining 4 pts were withdrawn for early disease progression, while 4 pts entered the $\mathrm{MF}$ and are still on treatment. TA at wk 12 showed partial response (PR) in $1 / 10$ or stable disease (SD) in 3/10 pts; TA at wk 24 showed PR and SD in $1 / 4$ and $3 / 4$ pts, respectively, with a clinical benefit (SD+PR) of $29 \%$. As previously reported for cutaneous MM, slow, steady decline in tumor volume and appearance of new lesions with subsequent shrinking has been observed. No grade $3 / 4 \mathrm{AE}$ and irAE were reported. Median overall survival is $32(2-52)$ wks.

Conclusions: Ipilimumab administration in pretreated uveal MM pts is feasible and safe. A sizeable proportion of treated pts experienced clinical benefit, with extended survival. These data, thought preliminary and in a limited number of pts, suggest that uveal MM might represent a promising indication for ipilimumab treatment to be further investigated.

\section{E16 GASTROINTESTINAL (GI) TRACT POORLY DIFFERENTIATED ENDOCRINE CARCINOMA (PDEC): MAMMALIAN TARGET OF RAPAMYCIN (MTOR) EXPRESSION ANALYSIS}

Laura Catena, Massimo Milione ${ }^{1}$, Antonino Carbone ${ }^{1}$, Marco Platania, Monika Ducceschi

${ }^{1}$ Francesca Dominioni, Lorenza Di Guardo, Roberto Buzzoni, Barbara Formisano and Emilio Bajetta. Medical Oncology Unit 2; ${ }^{1}$ Department of Pathology: Fondazione IRCCS Istituto Nazionale dei Tumori, Milano

Background: GI tract PDEC are rare tumours accounting 0.1\%-1\% of all GI malignancies. mTOR signalling pathway has emerged as a promising target for well differentiated endocrine carcinoma therapy. Because of different behaviour, PDEC are usually excluded from clinical trials employing the mTOR inhibitor RAD001 and actually their activity in PDEC is tested only in cell lines.

Objective: We conducted a retrospective analyses of PDEC in a mono-institutional series revised according to $\mathrm{WHO}$ classification and validated with

immunohistochemistry (IHC) for endocrine markers. We also aimed to testing if mTOR is expressed in the human PDEC.

Methods: Between 1984 until 2007, 640 NETs were evaluated in our Institution, of whom 36 (5.6\%) were diagnosed as PDEC (excluding SCLC). The staining for mTOR was optimised employing slides of normal kidney as positive control. To ensure antibody specificity, consecutive sections were incubated in the absence of primary antibody. In such cases no immunostaining was detected. The immunoreactivity was evaluated on a semiquantitative scale considering the extent (score: $0-4$ ) and the intensity (score: $0-3$ ) of staining. The product was used to obtain an immunostaining score (total score 0-12).

Results: There were 19M/17F, median age 59 years (range, 17-75). The primary site was: pancreas $12(34 \%)$, colon $6(19 \%)$, lung $6(19 \%)$, unknown $5(14 \%)$, small bowel 4 $(11 \%)$, others $3(10 \%)$. At diagnosis $30(83 \%)$ pts had Stage IV disease while $6(17 \%)$ underwent surgery. The overall survival was 18 months (range, 4-61+). mTOR expression is maintained at similar levels in $80 \%$ of samples, with no relationship with tumour origin, function, proliferation rate valued through MIB-1. 
Conclusions: In our series PDEC are more frequent and have a longer survival than in the literature. Our biological findings demonstrate expression of mTOR in human PDEC and support an extended analysis in order to understand the role of mTOR and the real activity of RAD001 in PDEC.

Partially supported by Fondazione Giacinto Facchetti O.N.L.U.S

\section{E17 ROLE OF MAIN CANDIDATE GENES (P16 CDKN2A, BRCA2, MC1R, NRAS, BRAF) IN SUSCEPTIBILITY AND PATHOGENESIS OF MELANOMA}

Giuseppe Palmieri', Antonio Muggiano ${ }^{2}$, Antonio Cossu ${ }^{3}$, Mario Budroni ${ }^{4}$, Corrado Caraco ${ }^{5}$, Elena Pagani ${ }^{6}$, Ignazio Stanganelli ${ }^{7}$, Sergio Canzanella ${ }^{8}$, MariaCristina Sini ${ }^{1}$, Milena Casula ${ }^{1}$

${ }^{1}$ Istituto di Chimica Biomolecolare, CNR, Sassari; ${ }^{2}$ Ospedale Oncologico Businco, Cagliari; ${ }^{3}$ Azienda Ospedaliero Universitaria, Sassari; ${ }^{4}$ Azienda Sanitaria Locale 1, Sassari; ${ }^{5}$ Istituto Nazionale Tumori Fondazione Pascale, Napoli; ${ }^{6}$ Istituto Dermopatico dell'Immacolata, Roma; ${ }^{7}$ Istituto Tumori Romagna, Meldola, Forli; ${ }^{8}$ Associazione House Hospital Onlus, Napoli; Italy

Background: Several genetic alterations have been demonstrated to contribute to development and progression of melanoma (melanomagenesis). In this study, we further investigated the impact of key-regulator genes in melanoma pathogenesis and susceptibility.

Methods: A large series of sporadic and familial cases $(\mathrm{N}=846)$ originating from South Italy was screened for germline mutations in $p 16^{C D K N 2 A}, B R C A 2$, and $M C 1 R$ candidate genes by DHPLC analysis and automated DNA sequencing. Melanoma cell lines $(\mathrm{N}=18)$ as well as paired primary melanomas and lymph node metastases from the same patients $(\mathrm{N}=35)$ were analyzed for somatic mutations in NRAS, BRAF, and p16 ${ }^{C D K N 2 A}$ genes.

Results: Mutually-exclusive mutations of NRAS and BRAF genes were observed at quite same rate (about two thirds) in cultured and in vivo melanomas (either primary or metastatic lesions). Conversely, $p 16^{C D K N 2 A}$ gene alterations were observed at increased rates moving from primary to metastatic melanomas and melanoma cell lines. No PTEN mutation was found in melanoma cell lines from our series.Activation of the RAS-RAF-ERK pathway was demonstrated to be due to a combination of molecular alterations (mainly, NRAS/BRAF mutations and $p 16^{C D K N 2 A}$ silencing). Regarding genetic predisposition to melanoma, $p 16^{C D K N 2 A}$ was found exclusively mutated in 16/545 (2.9\%) non-Sardinian patients, whereas BRCA2 germline mutations were observed in 4/91 (4.4\%) patients from North Sardinia only. Two MC1R germline variants, Arg151Cys and Asp294His, were significantly associated with melanoma in Sardinia.

Conclusions: Our findings further clarified that multiple molecular events are accumulating during melanomagenesis as well as that genetic factors predisposing to melanoma are geographically heterogeneous and strictly depending on patients' origin On this latter regard, prevalence of mutations in melanoma susceptibility genes needs to be evaluated within each specific geographical area.

\section{E18 CLINICAL AND IMMUNOHISTOCHEMICAL CHARACTERIZATION OF MULTIPLE PRIMARY MELANOMAS TO IDENTIFY FAMILIAL MELANOMA SYNDROME}

Giovanni Ponti ${ }^{1}$, Lorena Losi ${ }^{2}$, Giovanni Pellacani ${ }^{3}$, Annamaria Cesinaro², Elisa Boni $^{3}$, Patrizia Pepe ${ }^{3}$, Maria Teresa Landi ${ }^{4}$, Gabriele Luppi ${ }^{1}$, Pier Franco Conte ${ }^{1}$, Stefania Seidenari

${ }^{1}$ Department of Oncology and Haematology, University of Modena and Reggio Emilia, Modena, Italy; ${ }^{2}$ Department of Pathology, University of Modena and Reggio Emilia, Modena, Italy; ${ }^{3}$ Division of Dermatology, Department of Internal Medicine, University of Modena and Reggio Emilia, Modena, Italy; ${ }^{4}$ Genetic Epidemiology Branch, Division of Cancer Epidemiology and Genetics, National Cancer Institute, NIH, DHHS, Bethesda, MD,USA

Background: The development of Multiple Primary Melanomas (MPM) represents an intriguing phenomenon from both the clinical and the biomolecular point of view. MPM can arise in a patient either synchronously or metachronously and constitutes one of the criteria for identifying families with hereditary melanoma. p16 immunohistochemical analysis has been used to detect CDKN2A aberrations although its role as a preliminary screening test is debated. In this study, we evaluated the clinical, immunohistochemical and biomolecular features in a series of MPM patients.

Methods: Detailed personal and family history were obtained for each patient. Expression of p16, p53, B-catenin, Ecadherin, MLH1 and MSH2 genes was evaluated by immunohistochemistry (IHC). Constitutional mutations in the p16 gene were searched for by gene sequencing.

Results: 27 unrelated patients were identified who had been diagnosed with at least two primary melanomas. The median age at diagnosis was 54.08 years (range 21-94 years) and there were 16 males and 11 females. More than $30 \%$ of MPM patients had a positive family history of melanoma. In this familial group, the median age of malignant melanoma (MM) diagnosis was 50.02 years. Hereditary melanoma in patients with germline mutation L65P in the p16 gene shows disappearance of p16 staining from the nucleus, and weak immunoreactivity in the cytoplasm.
Conclusion: The relevance of MPM should be addressed in melanoma follow-up protocols and could be a useful marker for identification of Familial Melanoma Syndrome. The combined use of clinical features and p16 IHC analysis can be useful for the preliminary screening of multiple primary melanoma patients and recognition of the hereditary setting.

\section{E19 WELL-DIFFERENTIATED NEUROENDOCRINE TUMORS (NETS): ANALYSIS OF 86 CASES TREATED WITH LONG ACTING SOMATOSTATINE ANALOGUES (SSA)}

Mauro lannopollo, Miriam Ricasoli, lacopo Petrini, Monica Lencioni, Cinzia Orlandini, Sergio Ricci

U.O. Oncologia Medica Azienda Ospedaliero-Universitaria Pisana.

Background: Somatostatine analogues represent an important therapeutic choose for NETs. SSA have demonstrated activity in symptomatic control, but only few data in terms of time to progression (TTP) and overall survival (OS) have been reported. In pre-SSA era median OS was 26 and 67 months in foregut and midgut NETs respectively from start of treatment.

The aim of this analysis is to evaluate the outcome of patients treated with SSA. Materials and Methods: From June 1997 to January 2009, 86 pts with metastatic NETs were treated with SSA in our center. Patients characteristics were: median age 63 years (range 24-80); males 56, female 30; pancreatic 30, gastrointestinal 48, other sites 8; functioning 37 , non-functioning 49 ; symptomatic 51 ; metastatic disease at diagnosis 73; 77 pts had surgery; 76 patients had a positive Octreoscan; 79 pts had SSA as firstline therapy, 7 pts had previous therapy: IFN 2, s.c. Somatostatine 2, TACE 1, Radiometabolic therapy 1, Chemotherapy 1. All pts received SSA: Lanreotide 37, Octreotide 49

Results: Median TTP was 22 months (CI 95\% 16,5-27,5). Statistically significant differences were observed between: symptomatic and not symptomatic disease ( $24 \mathrm{vs}$ 17 months, $\mathrm{p}=0,05$ ); pancreatic, gastrointestinal and other sites (22 vs 23 vs 12 months, $\mathrm{p}=0,049)$.

Median OS was 113 months (C:I: 95\% 63-163); no statistically significant differences were observed among subgroups.

Conclusions: NETs have generally an indolent growth, but prognosis remains poor. This retrospective analysis suggests that SSA can improve the outcome of this disease. Further studies are required to identify biological markers for new combined therapies.

\section{E20 DERMATOFIBROSARCOMA PROTUBERANS (DFSP) AND FIBROSARCOMATOUS ('HIGH-GRADE”') DFSP: A MONO- INSTITUTIONAL RETROSPECTIVE ANALYSIS}

Emanuela Palmerini ${ }^{1}$, Marco Gambarotti ${ }^{2}$, Eric L Staals ${ }^{3}$, Gabriela Sieberova ${ }^{2}$, Alessandra Longhi ${ }^{1}$, Marilena Cesari ${ }^{1}$, Massimo E Abate ${ }^{1}$, Piero Picci ${ }^{4}$, Mario Mercuri ${ }^{3}$, Marco Alberghini ${ }^{2}$, Stefano Ferrari ${ }^{1}$

${ }^{1}$ Chemotherapy Unit; ${ }^{2}$ Surgical Pathology; ${ }^{3} 5^{\text {th }}$ Division of Orthopaedic Surgery; ${ }^{4}$ Laboratory of Oncologic Research, Istituto Ortopedico Rizzoli, Bologna, Italy

Background: DFSP and its fibrosarcomatous variant (FS-DFSP) are rare soft-tissue tumors accounting for $1,4 \%$ of all sarcomas. A retrospective analysis of our experience is reported.

Material and Methods: Patients with a diagnosis of DFSP and FS-DFSP performed at our institute between 1982 and 2009 were identified. Two pathologists reviewed the slides and agreed on the diagnosis. Demographic data, tumor related characteristics and treatments were collected.

Results: The study included 51 patients ( 22 female, 29 male); median age 43 years (range 7-91). Seventeen (33\%) had tumors in the lower limb, 15 (30\%) in the upper limb, 19 in the trunk $(37 \%)$. Eleven cases were pathology consultations and the survival analysis was made on 40 patients surgically treated at IOR. Previous surgical treatments (from 1 to 5, median 1) were documented in $36(90 \%)$ patients. Thirty-seven (92\%) patients had localized disease and $3(8 \%)$ were had metastases. 39 patients underwent surgery whereas 1 patient refused an amputation. A fibrosarcomatous variant was detected in 13 (32.5\%) patients. Adequate surgical margins were reported in $73 \%$ of patients. Radiotherapy (RT) was added in 11 patients ( 7 with FS-DFSP). Ifosfamide and/or doxorubicin-based chemotherapy was administered to 3 patients with FS-DFSP. Two patients were lost to follow-up. Incidence of local recurrence was $42 \%$ in FS-DFSP and $15 \%$ in DFSP. Three FS-DFSP patients developed distant metastases whereas none of DFSP did. With a median follow-up of 48 months (1-184), the 5-year overall survival (OS) was $93 \%$. The 5-year event-free survival (EFS) was $69 \%$ in localized patients. Histology (DFSP 65\% vs. FS-DFSP 38\%, p = 0.014), surgical margins (adequate $74 \%$ vs. inadequate $56 \%, \mathrm{p}=0.032$ ) and site (limb $49 \%$ vs. trunk $100 \%$, $\mathrm{p}=0.02)$ influenced the 5 -year EFS, whereas sex, use of RT or number of previous operations did not.

Conclusions: Patients with DFSP have a high survival probability. Site, adequate surgical margins and presence of the fibrosarcomatous component influence the local and metastatic recurrence. 


\section{E21 PROGNOSTIC VALUE OF MGMT METHYLATION STATUS IN PATIENTS WITH 1P/19Q INTACT ANAPLASTIC GLIOMAS}

Enrico Franceschi ${ }^{1}$, Alicia Tosoni ${ }^{1}$, Mario Ermani ${ }^{2}$, Carmelo Sturiale ${ }^{3}$, Patrizia Dall'Occa ${ }^{1}$, Annalisa Pession ${ }^{4}$, Enrico Di Oto ${ }^{4}$, Luca Morandi ${ }^{4}$, Stefania Bartolini ${ }^{1}$, Alba A Brandes

${ }^{1}$ Medical Oncology Department, Bellaria-Maggiore Hospital, Azienda USL of Bologna, Bologna, Italy; ${ }^{2}$ Neurosciences Department, Statistic and Informatic Unit, Azienda Ospedale-Università, Padova, Italy; ${ }^{3}$ Neurosurgery Department, Bellaria-Maggiore Hospital, Azienda USL of Bologna, Bologna, Italy; ${ }^{4}$ Pathology Department, Bellaria-Maggiore Hospital, Azienda USL of Bologna, Bologna, Italy

Background: Chromosomes $1 \mathrm{p} / 19 \mathrm{q}$ codeletion has been recognized as a prognostic and predictive factor in patients (pts) with anaplastic gliomas (AG). Non-codeleted (intact) anaplastic oligodendroglioma showed a survival comparable to that usually observed in pts with anaplastic astrocytomas; MGMT methylation status, moreover, has been found to be a prognostic factor in glioblastoma and anaplastic gliomas.

Methods: A retrospective analysis was made using a database of 253 AG pts followed prospectively between $01 / 1998$ and $11 / 2008$. We evaluated only pts who met the following inclusion criteria: age $\geq 18$ years; PS $0-2$; histological diagnosis of AG with $1 \mathrm{p} /$ $19 \mathrm{q}$ intact, as determined by FISH analysis; treatment with postoperative radiotherapy (RT) and chemotherapy (CT); MGMT status determined using methylation specific PCR. The study aim was to evaluate the role of MGMT methylation status in $1 \mathrm{p} / 19 \mathrm{c}$ codeleted AG pts. The log-rank test was used to evaluate the significance of the prognostic variables.

Results: 75 pts (m/f: 39/36, median age: 40 years, range: $18-70$ years) were enrolled. Histology was anaplastic oligodendroglioma in $19 \mathrm{pts}$, anaplastic oligoastrocytoma in 22 pts and anaplastic astrocytoma in 34 pts; all these pts were $1 \mathrm{p} 19 \mathrm{q}$ intact and received surgery, RT and CT. MGMT status, assessable in 66 pts $(88 \%)$, was methylated in 38 pts $(57.6 \%)$ and unmethylated in 37 pts (49.3\%). Median progression-free survival (PFS) was 27 months (95\%CI: $13.1-40.9)$. In multivariate analysis, no enhancement at time of diagnosis $(\mathrm{p}=0.03)$ and gross total resection $(\mathrm{p}=0.04)$ were significantly correlated with better PFS. Median survival was 74 months (95\%CI: 55.9-92.1). In multivariate analysis, only age $(p=0.005)$ and KPS $(\mathrm{p}=0.045)$ correlated with a better survival.

Conclusions: MGMT methylation status does not seem to provide adjunctive prognostic information in pts with $1 \mathrm{p} / 19 \mathrm{q}$ intact AG.

\section{E22 DECREASED LEVELS OF REGULATORY T (TREG) CELLS IN PERIPHERAL BLOOD OF MELANOMA PATIENTS AFTER TREATMENT WITH INTRAVENOUS HIGH DOSE INTERFERON (HDI)}

Ascierto PA, Napolitano M, Celentano E, Simeone E, Daponte A, Gentilcore G, Capone M, Palmieri G, Castello G, Mozzillo N

National Cancer Institute, Naples - Institute of Biomolecular Chemistry-CNR, Sassari

Background: Natural T regulatory (Treg) cells are deputed to controlling autoimmunity through "dominant tolerance" and represent approximately 5-10\% of the total CD4+ T cell population. They are expressing high levels of surface CD25 (high-affinity IL-2a receptor subunit), CTLA-4, and glucocorticoid-induced tumor necrosis factor-a receptor (GITR). Tregs have been shown to be present in tumor and tumor-draining lymph nodes, acting as a potential inhibitory population blocking or "balancing" effector cell function. Thus, depletion of Tregs or blockade of Treg function might be able to enhance antitumor immunity. Recent evidence has been reported about the possibility of High Dose Interferon (HDI) to act through an indirect immunomodulatory rather than a cytotoxic mechanism: a) correlation with the development of autoimmunity (Gogas, NEJM 2006); b) endotumoral increase of CD11c+/CD3+ cells and decrease of CD83+ cells in clinical responders. Therefore, we started a study to verify if iv HDI treatment in melanoma patients could be able to reduce the number of Treg cells in peripheral blood.

Patients and Methods: Analysis was performed on melanoma patients referring to the National Cancer Institute of Naples since July 2006 and who addressed to Neoadjuvan or Adjuvant treatment with iv HDI (20 million units $/ \mathrm{m}^{2}, 5$ days per week) for 4 weeks. Peripheral blood mononuclear cells (PBMC) were obtained from 22 consecutive melanoma patients. Blood draw was performed at days 0, 8, 15, 22 and 29. PBMC were thawed and labeled with anti-CD4-PerCP and anti-CD25-Pe (IL-2R1) (BD, San Diego, CA) and anti-FoxP3-FITC (PCH101) (eBioscience, San Diego, CA). Labeled cells were analyzed using a FACScalibur (Becton Dickinson).

Results and Discussion: Fifteen $(68.2 \%)$ out of 22 patients showed a decrease of Treg cells in peripheral blood. The average value at day 0 for circulating Treg cells (cTreg) was $2.7 \%$. The average percentage at day 29 was $1.4 \%$. The average reduction was 1,4 (50\% reduction in the average value of cTreg). Statistical analysis showed an average decrease of $0.29 \%$ per week of treatment. Despite of this clear trend in reducing cTreg by HDI induction treatment, statistical significance was not reached (probably due to the power of the study). Moreover, it has been observed great differences between the disease status, the prognosis (recurred/not recurred pts, alive/deceased) and an increased basal percentage of cTreg in PBMC. Our preliminary data are consistent for an effect of HDI on reducing circulating Treg cells, although no conclusion about the role of such reduction in terms of response to treatment or as prognostic markers of better/worse disease can be inferred. Further data are awaited in order to verify if the
Treg reduction after a HDI treatment may indeed contribute to the antitumor response.

\section{E23}

\section{GLIOMATOSIS CEREBRI: A SINGLE INSTITUTION REPORT}

Bertolini $F^{1}$, Depenni $R^{1}$, Fontana $A^{1}$, Falasca $A^{4}$, Meletti $S^{5}$, Valentini $A^{4}$, Giacobazzi $P^{2}$, Cavalleri $F^{6}$, Zunarelli $E^{3}$, Pinna GP $^{4}$

Oncology; ${ }^{2}$ Radiotherapy; ${ }^{3}$ Pathology section - Azienda OspedalieroUniversitaria - Policlinico di Modena; ${ }^{4}$ Neurosurgery; ${ }^{5}$ Neurology;

Neuroradiology - Azienda Sanitaria Locale di Modena, Nuovo Ospedale Civile S.Agostino Estense

Gliomatosis cerebri (GC) is defined as a diffuse neoplastic glial cell infiltration of the brain involving more than two cerebral lobes. Diagnosis and management of GC are difficult. Because of its diffuse nature, surgery is not suitable and large field radiotherapy carries the risk of severe toxicity.

Between May 2006 and February 2008, 6 patients (pts) (M-F=3-3; median age at diagnosis: 69 years; range: 44-74) were diagnosed with GC based on radiological characteristics and histological findings at University of Modena and Reggio Emilia. The aim of this retrospective analysis is to study clinical characteristics, the role of temozolomide and outcome of these pts. The analysis was performed using clinical charts, radiological investigations and pathological examinations.

Five pts underwent a stereotactic biopsy (positive for neoplastic cells in 2 on 5 cases), while only 1 pt was submitted to surgery (partial exeresis). Based on it, histological specimen was diagnostic in 3 on 6 pts and it was as follows: 1 low grade astrocytoma (G1-2), 1 high grade astrocytoma (G3) and 1 low grade oligoastrocytoma (G2). In 3 pts the diagnosis was only on neuroradiologic investigations. Four pts received an antineoplastic treatment for GC (66\%). One pts $(16 \%)$ was submitted to whole brain radiotherapy (54 Gy) and subsequent chemotherapy with temozolomide, while 3 pts $(50 \%)$ received chemotherapy with temozolomide. The median number of cycles delivered was 5 (range: 2-7). Only one pt developed a grade 1 hematologic toxicity (the one who received also radiotherapy). Median time to tumor progression was 6 months (range: 2-20), with a median overall survival of 16 months.

Survival data are similar to the literature. In this setting temozolomide is well tolerated and these results indicate that radiotherapy to extensive brain regions can be deferred until progressive disease is observed.

\section{E24 PHASE I STUDY OF NON-PEGYLATED LIPOSOMAL DOXORUBICIN IN COMBINATION WITH IFOSFAMIDE IN ADULT PATIENTS WITH METASTATIC SOFT TISSUE SARCOMAS}

Elisa Stroppa ${ }^{1}$, Alexia Bertuzzi ${ }^{1}$, Gabriele Di Comite ${ }^{1}$, Silvia Salamina ${ }^{1}$, Alfredo Barbato ${ }^{2}$, Armando Santoro ${ }^{1}$

${ }^{1}$ Dipartimento di Oncologia ed Ematologia, Istituto Clinico Humanitas, Rozzano (MI): ${ }^{2}$ Cephalon Srl, Roma

Aim: To evaluate the maximum tolerated dose (MTD) and safety of the combination of non- pegylated liposomal doxorubicin (Myocet) and ifosfamide in patients with metastatic soft tissue sarcomas.

Methods: Cohorts of four patients with metastatic soft tissue sarcomas received up to five cycles of intravenous ifosfamide $3000 \mathrm{mg} / \mathrm{m}^{2}$ on days $1-3$ in combination with escalating doses of intravenous Myocet on day 1 every 3 weeks until dose limiting toxicity (DLT) in at least one patient. Myocet was planned to be escalated through $10 \mathrm{mg} / \mathrm{m}^{2}$ increments / cohort from 40 to $80 \mathrm{mg} / \mathrm{m}^{2}$. Toxicity was graded according to the National Cancer Institute Common Toxicity Criteria v3.0.

Results: Ten patients were enrolled in the study and 8 of them received the treatment Median age was 45 years, 5 patients were female. Two patients had received 1 cycle of prior chemotherapy. DLT, consisting of grade IV neutropenic fever, was reached in one patient at dose cohort $2\left(50 \mathrm{mg} / \mathrm{m}^{2}\right)$.

Conclusion: The MTD of Myocet, given in combination with ifosfamide $9000 \mathrm{mg} / \mathrm{m}^{2}$, is $40 \mathrm{mg} / \mathrm{m}^{2}$. A prospective study to define the activity of this combination is ongoing.

\section{E25 NEW SCHEDULE OF FOTEMUSTINE IN TEMOZOLAMIDE-} PRETREATED PATIENTS WITH RELAPSING GLIOBLASTOMA

Raffaele Addeo ${ }^{1}$, Serena De Santi $\mathrm{M}^{2}$, Liliana Montella ${ }^{1}$, Patrizia lodice ${ }^{1}$, Ciro Parlato ${ }^{3}$, Vincenzo Faiola ${ }^{1}$, Gregorio Cennamo ${ }^{1}$, Rosario Guarrasi ${ }^{1}$, Salvatore Del Prete ${ }^{1}$

${ }^{1}$ U.O.Complessa Di Oncologia Frattamaggiore (NA); ${ }^{2}$ U.O. Complessa Neurochirurgia Nocera Inferiore (SA); ${ }^{3}$ Dipartimento di Neurochirurgia S.U.N. Napoli

The present study aims to define the feasibility and the effectiveness of a new biweekly schedule of Fotemustine in patients with recurrent Glioblastoma, after at least a previous treatment. Primary endpoint was progression-free survival at 6 months, other secondary objectives were: clinical response, overall survival, disease free survival and toxicity. Forty-one patients (median age: 53 years; median Karnofsky performance status at progression: 80 ) underwent a second-line chemotherapy with 
Fotemustine. Selected patients were previously treated with a standard Radiotherapy course with concomitant Temozolamide. After MRI- proven tumor relapse or progression, all patients underwent chemotherapy with Fotemustine, given intravenously $80 \mathrm{mg} / \mathrm{m}^{2}$ every two weeks, for 5 consecutive administrations (induction phase), and then every 3 weeks at $100 \mathrm{mg} / \mathrm{m}^{2}$ as maintenance. Toxicity grading was based on the National Cancer Institute's Common Criteria (version 2.0). A total of 338 infusions were administered; the median number of cycles administered was 8 . All patients completed the induction phase and 29 patients received at least one infusion of maintenance. Response to treatment was assessed on MacDonald criteria. One complete response $(2,4 \%)$, nine partial responses $(22 \%)$ and 17 cases of stable disease $(41 \%)$ were observed. Median time to progression was 6.3 months. Progression free survival at 6 months was $53.6 \%$. Median survival from the beginning of Fotemustine chemotherapy was 10.2 months. The schedule was generally well tolerated, and the main toxicities seen were hematologic (grade 3 thrombocytopenia: in 2 cases). To the best of our knowledge, this is the first report specifically dealing with the use of biweekly induction schedule of Fotemustine. The study demonstrates that Fotemustine has therapeutic efficacy as single-drug secondline chemotherapy with a favorable safety profile.

\section{E26 NEMO (NF-KB ESSENTIAL MODULATOR) BINDING DOMAIN (NDB) PEPTIDE COULD INDUCE APOPTOSIS IN SEVERAL MELANOMA CELL LINES}

De Maio A, Capone M, Natale E, Gentilcore G, Gravina C, Mistretta C, Di Martino S, Simeone E, Daponte A, Caracò C, Palmieri G, Faraone-Mennella MR, Ascierto PA

National Cancer Institute, Naples - Department of Biochemical and Biochemistry "Federico II" University, Naples - Institute of Biomolecular Chemistry-CNR, Sassari

Background: Recent evidences indicate that activation of nuclear factor-kappa B (NF$\mathrm{kB}$ ) could play a role in melanoma progression. NF-kB is able to regulate the expression and the function of a wide spectrum of genes involved in cell cycle control, apoptosis, cell growth, tissue invasiveness and inflammation. For this reason NF-kB is persistently activated in many types of human tumors, protects developing tumor cell from death and thereby contributes to tumorigenesis and cancer therapy resistance (Lee $\mathrm{CH}$ et al. 2007). NF-kB is anchored and blocked outside the nucleus, under physiological conditions, by a family of cytoplasmic inhibitors, IKB. NF-kB is activated by kB kinase complex (IKK), consisting of two catalytic subunits, IKK alfa and IKK beta, and a regulatory component, IKK gamma, also named NF-kB Essenzial Modulator (NEMO) (Karin M 2006). Recent advances revealed that a very small region in the $\mathrm{COOH}$ terminus of IKKalfa, (L738-L743), and IKKbeta (L737-L742), was essential for stable interaction with NEMO, and for the assembly of the IKK-NEMO complex. The identification of the NEMO- binding domain (NBD) peptide that can block the activation of the IkB kinase (IKK) complex, have provided the possibility to selectively modulate the activation of NF-kB by targeting the NBD-NEMO interaction. NBD peptides inhibited the interaction of IKKb with NEMO in vitro and prevented formation of the endogenous IKK complex. Recently, we demonstrated that the administration of NBD peptide in A375 cells line produces inhibition of constitutive NF-kB DNA-binding activity and inhibition of IKK activity. We also shown that NBDpeptide inhibits A375 cell growth, inducing apoptosis by activation of caspase-3, as confirmed the cleavage of poly(ADPribose) polymerase (PARP-1), the best marker of this process ( Ianaro A. et all, 2008).

Aim of actual research is to investigate the effect of the NBD-peptide on NF-kB activity and survival of different melanoma cells line (397, SK-Mel 28, B16-F10, M14 ), which present different mutations in several genes.

This confirms a possible role of NF-kB in melanoma progression and, furthermore, blocking NF-kB activity it's possible to induce apoptosis in melanoma cell lines. The administration of NBD peptide could modulate NF-kB activity which could be critical for melanoma cell survival in melanoma cell lines and could offer new possible therapeutic strategies for melanoma.

\section{E27 PRELIMINARY ANALYSIS OF THE GENE EXPRESSION PROFILE OF JAGGED/NOTCH SIGNALING PATHWAY COMPONENTS IN HUMAN THYROID CARCINOMAS}

Annamaria Piscazzi ${ }^{1}$, Annarita Fabiano ${ }^{2}$, Anna Farese ${ }^{2}$, Francesca Maddalena ${ }^{1}$, Eleonora Costantino ${ }^{1}$, Alberto Fersini ${ }^{3}$, Mauro Cignarelli ${ }^{2}$, Matteo Landriscina ${ }^{1}$ ${ }^{1}$ Clinical Oncology Unit; ${ }^{2}$ Endocrinology and Metabolic Disease Unit, Department of Medical Sciences; ${ }^{3}$ General Surgery Unit, Department of Surgical Sciences, University of Foggia, Viale Pinto 1, 71100 Foggia, Italy

Background: Jagged/Notch signaling plays a pivotal role in the regulation of many fundamental cellular processes such as proliferation, stem cell maintenance and differentiation. There is increasing evidence that this signaling pathway is involved in human carcinogenesis and exerts oncogenic or tumor suppressor effects depending on the level of signaling activity and the context in which it occurs. The role of Jagged/ Notch signaling in thyroid carcinogenesis started recently to be elucidated. Indeed, it has been suggested that components of Jagged/Notch pathway are expressed in human thyroid follicular cells and are regulated by TSH. Interestingly, Notch receptors seem to be down-regulated only in undifferentiated thyroid carcinomas and the reestablishment of Notch activity correlates with reduced cell proliferation, increased NIS expression and gain in cell differentiation, suggesting a tumor suppressor function for Notch pathway. These findings appear to be in conflict with the evidence that Jagged 1 expression and Notch activity is increased in stem cells obtained from human thyroid cancer cell lines, suggesting rather an oncogenic role.

Material and Methods: We evaluated, by quantitative RT-PCR, the gene expression profile of Notch receptors 1-4 and their ligands Jagged 1 and 2 in a series of 10 human thyroid carcinomas in comparison to the respective non-infiltrated thyroid gland and in poorly-differentiated thyroid carcinoma FRO cells in comparison to welldifferentiated papillary thyroid TPC-1 cells. Gene expression levels of specific genes were interpreted as up-regulated if found increased at least 2 times.

Results: Preliminary results from this limited series of thyroid carcinomas revealed the up-regulation of Notch 1 and 2 gene expression in, respectively, 7/10 and 5/10 thyroid tumors, and the up-regulation of Jagged 1 and 2 gene expression in, respectively, $3 / 10$ and $9 / 10$ thyroid tumors. No major change in the gene expression profile of Notch 3 and 4 were observed. Consistently, poorly-differentiated thyroid FRO cells exhibited increased expression of Notch 1 and 2 genes, as well as up-regulation of Jagged 1 and 2 expression compared to well-differentiated papillary thyroid tumor TPC-1 cells.

Conclusions: These findings suggest that Jagged/Notch components may exert an oncogenic role in thyroid carcinogenesis, even though further studies are needed to better define their function in the loss of cell differentiation and in the epithelialto-mesenchymal transition of thyroid tumors.

\section{E28 COMPARATIVE STUDY OF QUALITY OF LIFE (QOL) IN PATIENTS AFFECTED FROM SARCOMA AND NON SARCOMATOUS ONCOLOGICAL DISEASES}

Dal Canton O, Lombardi I, Comandone A, Bergnolo P, Oliva C, Boglione A, Chiadò Cutin S, Pochettino P, Inguil M, Garetto F, Bianco L, Berno E., Giubellino E Dept. Medical Oncology Ospedale Gradenigo, Gruppo Piemontese Sarcomi Italian Sarcoma Group

Introduction: The importance of QoL issues for cancer patients is well recognized either by clinicians or by patients and their families. Over the past several decades numerous studies have addressed the investigation on physical, emotional, social wellbeing of cancer patients mainly during the period of active oncological care.

Aim of this study was the QoL in pts with soft tissue and bone sarcomas compared to the patients with different oncological diseases, in order to clarify the possible differences in psychological attitude and coping.

Patients and method: QoL was assessed with Functional Assessment in Cancer Therapy-General and Hospital Anxiety and Depression Scale questionnaires. FACT-G has 34 items investigating physical, functional, emotional and social/family well-being. HADS scale was created as screening instrument to evaluate anxiety and depression symptoms in hospital patients; it consists of 2 subscales and 14 items. From January 2007 to December 2008 the questionnaires were administered in the 2 different groups: 42 pts with sarcomas and 42 patients with common tumours (breast, colon). All pts were under active chemothepy.

Comparison between the groups was performed by K Cohen system.

Results: The 2 groups were well balanced in age, sex, PS and stage of disease. Therapies were distinguished between adjuvant and palliative settings.

The 2 groups recorded similar results in agreement of the patient-doctor relationship (52\% sarcomas vs $51 \%$ other tumours), in familiar, social ( $50 \%$ vs $52 \%$ ) and functional satisfaction ( $57 \%$ vs $59 \%$ ).

Sarcoma patients did worst in anxiety (80\% vs $57 \%)$, depression ( $75 \%$ vs $61 \%$ ) and physical symptoms (64\% vs $70 \%)$.

Conclusion: Our comparative study put in evidence that sarcomas patients present a different coping during the active treatment of the disease.

The rarity of the disease, the lack of diffuse expertise among the clinicians, the need to move from far away to be treated in a Specialized Center added to the difficulties in movement and everyday activities can explain the worst results.

\section{E29 PINEOBLASTOMA IN ADULTS: A RETROSPECTIVE STUDY}

Mazzocchi V, Franceschi E, Tosoni A, Benevento F, Poggi R, LaTorre L, Dall'Occa P, Tomasello C, Maestri A, Brandes AA

Background: Pinealoblastoma (PB) is an extremely rare brain tumor in adults, and only occasional data are available about the treatment of these diseases.

Methods: A retrospective analysis of our brain tumors database was found to evaluate the management and outcomes of adults PB patients followed between January 1989 and December 2008. We evaluated only pts who met the following inclusion criteria: age $\geq 18$ years; PS $0-2$; histological diagnosis of $\mathrm{PB}$.

Results: nine patients were included into the analysis ( $\mathrm{m} / \mathrm{f}: 4 / 5$, median age: 33 , range: 18-44). Median follow up was 48 months (range: 20 - 147). Surgical resection was complete in 4 patients and partial in 5 patients. All but one patients showed positive cerebrospinal fluid. All the patients received whole brain and craniospinal irradiation. In patients with residual disease after surgery, radiotherapy showed 1 complete 
response (CR), 1 partial response (PR), and 3 disease stabilizations (SD). Cisplatinumetoposide based chemotherapy was administered as adjuvant treatment in 5 patients and at time of progression in 4 patients ( 1 CR, 2 SD and 2 progressions of the disease). All the patients experienced disease progression after chemotherapy, and median time to progression was 30.5 months (range: 2-69). At time of the analysis, 3 patients were alive. Median overall survival from time of surgical resection was 97 months $(95 \% \mathrm{CI}$ 14.3-179.7).

Conclusions: Adult PB is a rare and aggressive disease, that frequently recurs despite multimodal local and systemic treatments.

\section{E30 DOSE ESCALATED ACCELERATED INTENSITY MODULATED RADIATION THERAPY (IMRT) CONCURRENT WITH TEMOZOLAMIDE IN PATIENTS WITH GLIOBLASTOMA (GMB): A PHASE I STUDY}

Samantha Mignogna ${ }^{1}$, Franco Calista ${ }^{1}$, Rosa Tambaro ${ }^{1}$, Alessandro Cocciolo ${ }^{1}$, Caterina Montoro ${ }^{1}$, Marica Ferro ${ }^{1}$, Vincenzo Picardi ${ }^{1}$, Edy Ippolito ${ }^{1}$, Mariangela Massaccesi ${ }^{1}$, Liberato DI LULLO ${ }^{2}$, Divina Traficante ${ }^{2}$, Gianfranco Giglio ${ }^{3}$, Giustino Antuzzi $^{3}$, Antuzzi Luigi Sofo ${ }^{1}$, Alessio G Morganti ${ }^{1}$

${ }^{1}$ Dipartimento di Oncologia Università Cattolica Del Sacro Cuore, Centro di

Ricerca e Formazione ad Alta Tecnologia nelle Scienze Biomediche di

Campobasso; ${ }^{2}$ U.O. Oncologia Ospedale "F. Veneziale" Isernia;

${ }^{3}$ U.O. Oncologia Ospedale "Cardarelli" CampobassoTesto

Introduction: The primary objective of this study was to define the maximum tolerated dose (MTD) of escalating total doses of radiotherapy delivered concurrently with a daily fixed dose of temozolamide, within the same overall treatment time, after resection of GBM.

Methods and Materials: Patients with resected primary supratentorial GBM were enrolled. Chemotherapy with temozolamide was administered during $(75 \mathrm{mg} /$ day $)$ and for one year after IMRT (150-200 mg/day, for five consecutive days, every four weeks). IMRT targeted the residual tumor (if any) and the tumoral bed plus a $1 \mathrm{~cm}$ margin (Clinical Target Volume, CTV-1), the peri-tumoral edema plus a $2 \mathrm{~cm}$ margin (CTV2). The planning target volumes (PTV-1 and PTV-2) were obtained by adding a homogeneous $5 \mathrm{~mm}$ margin to CTVs. IMRT was delivered in 25 fractions, within an overall treatment time of 5 weeks. A total dose of $45 \mathrm{~Gy}$ was given to PTV-2. Radiation dose escalation was planned for PTV-1 only. The starting radiation dose was $60.0 \mathrm{G}$ within five weeks (2.4 Gy/fraction). Escalation was achieved up to a total dose of 65.0 Gy (2.6 Gy/fraction), by increasing the fraction size by $0.1 \mathrm{~Gy}$, while keeping constant the duration of IMRT to 5 weeks. Acute toxicity was graded according to RTOG/ EORTC criteria.

Results: Nineteen patients were enrolled (60.0 Gy:7 patients; 62.5 Gy: 6 patients, 65.0 Gy: 6 patients). After a median follow-up period of 23 months (range: $8-40$ month) no dose-limiting toxicities (acute Grade >or $=3$ irreversible central nervous system toxicities) were observed at any dose level. Central nervous system and cutaneous toxicities G1-2 were recorded in 11 and 9 patients, respectively.

Conclusions: The results of our study indicate that a total radiation dose up to $65.0 \mathrm{~Gy}$ (2.6 Gy/fraction) concurrently with temozolamide $(75 \mathrm{mg} / \mathrm{m} 2)$ can be safely administered to the tumoral bed or residual disease after resection of GBM, using IMRT. Phase two studies could be proposed in order to investigate the feasibility of this adjuvant treatment regimen.

\section{E31 VINCRISTINE, DOXORUBICIN, CYCLOPHOSPHAMIDE, ACTINOMYCIN D, IFOSFAMIDE AND ETOPOSIDE IN ADULT AND PEDIATRIC PATIENTS WITH NON METASTATIC EWING SARCOMA. FINAL RESULTS OF A MONOINSTITUTIONAL STUDY}

Stefano Ferrari, Emanuela Palmerini, Marco Alberghini, Eric Staals, Mario Mercuri, Enza Barbieri ${ }^{1}$, Alessandra Longhi, Laura Cantero, Piero Picci, Gaetano Bacci Istituto Ortopedico Rizzoli, Bologna. ${ }^{1}$ Istituto di Radioterapia, Università di Bologna

Background: To investigate a six-drug combination in adult and pediatric patients with non metastatic Ewing sarcoma (ES).

Methods: Alternating cycles of vincristine $1.5 \mathrm{mg} / \mathrm{m} 2$ (TOP $2 \mathrm{mg}$ ), doxorubicin $80 \mathrm{mg} /$ $\mathrm{m} 2$ and cyclofosfamide $1200 \mathrm{mg} / \mathrm{m} 2$ (week $0,6,13,22,31$ ), ifosfamide $9 \mathrm{~g} / \mathrm{m} 2$, vincristine $1.5 \mathrm{mg} / \mathrm{m} 2$ (TOP $2 \mathrm{mg}$ ) and actinomycin D $1.5 \mathrm{mg} / \mathrm{m} 2$ (TOP $2 \mathrm{mg}$ ) (week $3,16,25,34$ ), ifosfamide $9 \mathrm{~g} / \mathrm{m} 2$ and etoposide $450 \mathrm{mg} / \mathrm{m} 2$ (week 9,19,28,37) were administered. Primary chemotherapy-induced necrosis was graded: G3 (complete necrosis), G2 (microfoci of tumor cells) and G1 (macrofoci of tumor cells).

Results: From 1996 to 1999, 50 patients with ES were enrolled; median age 23.5 years (4-56 yrs). In adult and pediatric ( $<18$ years) patients the incidence of G4 leukopenia was $62 \%$ and $74 \%$ with febrile neutropenia in $13 \%$ and $21 \%$ respectively. G4 thrombocytopenia occurred in $3 \%$ of cycles in adults and in $7 \%$ in pediatric patients. Platelets and red blood cells transfusions were required in $1 \%$ and $11 \%$ of cycles in adults and in $6 \%$ and $24 \%$ of cycles in pediatric patients. Chemoinduced necrosis (in 28 patients) was G3 in 36\%, G2 in $21 \%$ and G1 in $43 \%$. With a median follow-up of 110 months (36-129) 5 -year OS and EFS were $72 \%$ and $66 \%$. The 5 -year EFS was $58 \%$ for adult patients and $79 \%$ for pediatric patients $(p=0.004)$. According to the histologic response, the 5-year EFS was $90 \%$ and $83 \%$ in G3 and G2, and $42 \%$ in G1 $(\mathrm{p}=0.02)$.

Conclusions: The 6-drug combination can be administered safely in adult populations. About $40 \%$ of patients have a poor chemoinduced tumor necrosis leading to poor probability of survival. New strategies are recommended to improve survival of poor responders to the 6-drug combination

\section{E32 A CASE REPORT OF FOLLICULAR THYROID CANCER SHOWING A REMARKABLE EFFECT OF SORAFENIB}

Caterina Viscomi, Mariagrazia Propato, Monica Ventura, Iole Cucinotto, Vito Barbieri, Angela Salvino, Maria Saveria Rotundo, Domenico Ciliberto, Pierfrancesco Tassone, Pierosandro Tagliaferri

Medical Oncology Unit, University of Magna Græcia, Catanzaro, Italy

This article is not included in your organization's subscription. However, you may be able to access this article under your organization's agreement with Elsevier.

Thyroid cancer is the most common malignancy of the endocrine system. Surgery is the standard option for radical treatment strategy. Recently, activation of the EGFR kinase pathway has been demonstrated to play a critical role in the pathogenesis of this disease. In the last years the EGFR pathway has been explored as therapeutic target for thyroid cancer. A number of compounds against this pathway have been studied and showed antitumor effects in preclinical studies. These agents are being tested in ongoing clinical trials.

Here we describe a 58-year-old woman with metastatic thyroid cancer who demonstrated a major response to Sorafenib. In June 2006 the patient underwent total thyroidectomy. In February 2007 and then in January 2008, following the detection of radioiodine hypercaptation at cervical multiple areas, she received radioiodine ablation. In April 2008, multiple pulmonary and bone metastases were detected by CTscan. She then underwent antiresorptive treatment with zoledronic acid and radiotherapy on the pelvis and cervical spine. In the absence of other available effective treatments for the metastatic disease, and in view of the striking biological rationale, the patient was then treated with Sorafenib $400 \mathrm{mg}$ bd. At the start of the treatment the tireoglobulin (TG) was $27944 \mathrm{ng} / \mathrm{ml}$; after one month of treatment (TG) was $17056 \mathrm{ng} /$ $\mathrm{ml}$, and after two months $11866 \mathrm{ng} / \mathrm{ml}$. Sorafenib demonstrated a remarkable effect on both the bone and lung lesions. Indeed CT showed significant reduction of lung disease (PR) with clinical benefit in terms of bone pain. This treatment lasts 5 months from the beginning, and is well tolerated.

The present report indicates that treatment with Sorafenib is an effective option for thyroid follicular carcinoma which needs to be evaluated in clinical trials.

\section{E33 LONG-ACTING ACTIVITY OF TAXANE-BASED CHEMOTHERAPY IN HEAVILY PRE-TREATED SARCOMA PATIENTS}

Giuseppe Pasini ${ }^{1}$, Cinzia Possenti ${ }^{1}$, Stefania Nicoletti ${ }^{1}$, Manuela Fantini ${ }^{1}$, Fabrizio Drudi $^{1}$, Davide Tassinari ${ }^{1}$, Emiliano Tamburini ${ }^{1}$, Lorenzo Gianni ${ }^{1}$, Maximilian Papi ${ }^{1}$, Oliverio Giovanni', Alberto Ravaioli

Ospedale Infermi Rimini, U.O. Oncologia ed Oncoematologia

The number of effective drugs for the treatment of adult metastatic soft tissue sarcomas is very low. First line treatment of most high grade soft tissue sarcomas is based on the use of anthracyclines with or without ifosfamide. At disease progression the options are few and the prognosis is poor.

We report two cases of long-term response to combination chemotherapy with paclitaxel and pegylated liposomal doxorubicin administered sequentially to docetaxel and gemcitabine in patients with metastatic soft tissue sarcomas heavily pretreated.

The first patient was a 38 year old woman, who had already undergone surgery and four regimens of chemotherapy for metastatic leymiosarcoma of the uterus; the patient received gemcitabine days $1-8,900 \mathrm{mg} / \mathrm{m}^{2}$ and docetaxel day $8,100 \mathrm{mg} / \mathrm{m}^{2}$ q21 until disease progression. After fourteen cycles we started paclitaxel day $1,60 \mathrm{mg} /$ $\mathrm{m}^{2}$ in combination with pegylated liposomal doxorubicin day $2,20 \mathrm{mg} / \mathrm{m}^{2}, \mathrm{q} 15$ for fourteen cycles. The patient is at present well and is continuing this therapeutic regimen.

The second patient was a 50 year old man, with metastatic liposarcoma, who had undergone surgery and three previous chemotherapeutic regimens. We administered paclitaxel day $1,60 \mathrm{mg} / \mathrm{m}^{2}$, in combination with pegylated liposomal doxorubicin day $2,20 \mathrm{mg} / \mathrm{m}^{2}, \mathrm{q} 15$ until disease progression. After twelve cycles, we performed docetaxel day $1,70 \mathrm{mg} / \mathrm{m}^{2}$ in combination with gemcitabine days $1-8,900 \mathrm{mg} / \mathrm{m}^{2} \mathrm{q} 21$. After fifteen cycles the disease progressed. For both regimens no haematologic nor nonheamatologic G3-4 toxicities were observed. (prophylactic G-CSF was used in both patients). Patients received treatment for long time, with low-mild toxicity, improvement of symptoms and a good quality of life.

In conclusion taxanes sequentially administered in combination with gemcitabine or pegylated liposomal doxorubicin and in particular paclitaxel in combination with pegylated liposomal doxorubicin at low dosage can be active and well tolerated in the treatment of heavily pretreated metastatic sarcomas. 
E34 PHASE II TRIAL OF INDUCTION STANDARD DOSE OF TEMOZOLAMIDE FOLLOWED BY TEMOZOLAMIDE AND CONCURRENT RADIOSURGICAL TREATMENT IS EFFECTIVE TREATMENT IN SINGLE BRAIN METASTASIS FROM SOLID TUMORS

Pace $R^{1}$, Del Matto $N^{1}$, Santarelli $M^{2}$, Rauco $A M^{1}$, De Santis $G^{1}$, Capparella ${ }^{1}$, Domini $\mathrm{P}^{3}$, Lugini $\mathrm{A}^{1}$

"Medical Oncology, Ospedale Generale "San Camillo de Lellis", Rieti, Italy;

'Division of Radiotherapy, Ospedale Generale "San Camillo de Lellis", Rieti,

Italy; ${ }^{3}$ Medical Oncology, Ospedale Palombara Sabina (Roma), Palombara Sabina, Italy

Background: Brain metastases occur in approximately $40 \%$ of all cancer patients with metastatic disease and are associated with a poor prognosis. Many patients can presen a progression of disease in the brain with single lesions, which do not always determine the onset of neurological symptoms. Surgery or localized radiosurgery (SRS) is suggested as an accepted therapeutic option for those patients who have only a single brain lesion. Temozolamide is oral alkylating agent with a good safety profile which crosses the blood brain barrier, reaching an effective concentration in the CNS, and has shown activity against many solid tumors.

Patients and Methods: Objective of the study is to assess the efficacy and safety of induction therapy with orally temozolamide at a dose of $250 \mathrm{mg} / \mathrm{sqm}$ gg.1-5, followed after 2 weeks of combined treatment of orally temozolamide $100 \mathrm{mg} / \mathrm{sqm}$ continued with stereotactic radiotherapy (total dose $2500 \mathrm{cGy}$ for 5 days), followed subsequent 4 cycles orally temozolamide $250 \mathrm{mg} / \mathrm{sqm}$ gg. $1-5$ every 4 weeks. From 06/2008 to 02/ 20096 cancer patients (pts) with metastatic single brain metastasis less than $3 \mathrm{~cm}$ in diameter received this treatment. Median age was 62 years, and PS was $0-1,2$ and 3 in 2, 3 and 1 patients. NSCLC/breast cancer respectively $3 / 3$.

Results: All patients were evaluable for toxicity and for response. Toxicity was: grade 1 thrombocytopenia (50\%); grade 1 asthenia (33\%), grade 1 anemia ( $33 \%$ ). 3/6 patients experienced RC; $2 / 6$ patients experienced SD.

Conclusions: The results of this study show that the combination is highly efficacious: improving survival and quality of life. These results confirm the effectiveness and the good profile of toxicity of the combination treatment.
E35 INTEGRATED REGIMEN THERAPEUTIC FOR MELANOMA ADVANCED

Dr G.Giarratano*, Dr Emilio Triglia*, Dr Giovanni Scuderi**, Dr Liborio di Cristina*** Dr Stefano Vitello*

*U.O. Oncologia Medica Osp. S. Elia Caltanissetta; **U.O. Dermatologia

Osp.S.Elia Caltanissetta; ${ }^{* * *} U$.Oncologia alcamo (TP)

Advanced in public awareness, surgical techniques, and adjuvant therapy have improved outcomes for patients with melanoma; however, it remains a highly morbid disease.

In fact the systemic therapy of advanced melanoma is one the most frustating task for the medical oncologist: the treatment yields few durable remission and minimal impact on survival $(<6$ months).

The response rate to $50-60 \%$ with integrated treatment, but with more toxicity than chemotherapy regime alone.

In our structure we have adopted an integrated regimen therapeutic(polybiologicchemotherapy), repeated every 28 days:

\begin{tabular}{llll} 
- Fotemustine & $100 \mathrm{mg} / \mathrm{m} 2$ & e.v. & days 1,$8 ;$ \\
-Cisplatin & $40 \mathrm{mg} / \mathrm{m} 2$ & e.v. & days 1,$2 ;$ \\
-Interleukin-2 & 6.000 .000 & s.c. & days $4,5,6,16,17,18 ;$ \\
-Interferon-alfa & 6.000 .000 & i.m. & days $8,10,12,14 ;$ \\
- Interferon-alfa & 3.000 .000 & i.m & days $4,6,16,18$. \\
\hline
\end{tabular}

We treated 21 patients(pts):14 men/7 women, median age 64 (range 31-68): with metastases to sites visceral( $10 \mathrm{pts})$;

with metastases to site no visceral (6 pts); with unresectable brain metastases ( 3 pts); with metastases visceral and brain ( 4 pts). After $4-6$ cycle of therapy the results are: 9 R.O.

whose 3 in patients with metastases visceral, 4 in patients with metastases no visceral, 2 in patients with brain metastases);

5 S.D.

The most common side effects were vague symptoms, such malaise, fatigue, chills and fever, in any case limited to WHO grades 1 and 2 .

None of the patients required an interruption in treatment or was hospitalised during the treatment period.

The duration of response has been $>8$ months, with range $5-15+$

The encouraging results incit we to continue the study 